\title{
Comparing swimsuits in 3D
}

\author{
Erik van Geer ${ }^{\mathrm{a},}$, Johan Molenbroek ${ }^{\mathrm{b}}$, Sander Schreven ${ }^{\mathrm{c}}$, Lenneke deVoogd-Claessen ${ }^{\mathrm{d}}$, \\ Huib Toussaint $\mathrm{c}^{\mathrm{c}}$ \\ ${ }^{\mathrm{a}}$ Erik van Geer Product Development, the Netherlands \\ ${ }^{\mathrm{b}}$ Applied Ergonomics \& Design, Industrial Design Engineering, TU Delft,j.f.m.molenbroek@tudelft.nl, Delft, The \\ Netherlands \\ ${ }^{c}$ Human Movement Sciences, VU University Amsterdam, the Netherlands \\ ${ }^{d}$ Sport Innovation, TU Delft
}

\begin{abstract}
In competitive swimming, suits have become more important. These suits influence friction, pressure and wave drag. Friction drag is related to the surface properties whereas both pressure and wave drag are greatly influenced by body shape. To find a relationship between the body shape and the drag, the anthropometry of several world class female swimmers wearing different suits was accurately defined using a 3D scanner and traditional measuring methods. The 3D scans delivered more detailed information about the body shape. On the same day the swimmers did performance tests in the water with the tested suits. Afterwards the result of the performance tests and the differences found in body shape was analyzed to determine the deformation caused by a swimsuit and its effect on the swimming performance. Although the amount of data is limited because of the few test subjects, there is an indication that the deformation of the body influences the swimming performance.
\end{abstract}

Keywords: 3D measuring, anthropometry, swimming

\section{Introduction}

In the past few years swimsuits have been subject of discussion because of their influence on swimming performance. Media were unsure why the new generation of swimsuits performed better than older ones.

Often buoyancy was mentioned as the cause for higher performance because the swimmers felt like they were floating higher in the water. Having Archimedes' principle in mind we feel that this cannot account for $100 \%$ of the differences in performance that occurred, leaving the way open for other theories, including body deformation, that explain the increase in performance of swimmers wearing such suits.

The speed of a swimmer is essentially the difference between propulsion and drag. Since the suits do not contribute in propulsion of the swimmer drag seems to be leading. For a better explanation of the principles behind the high performance suits more research was needed.

\section{Literature}

Swimming competitions are held with different disciplines; this project focuses on front crawl for female swimmers in an indoor competition. During a competition, a race can be divided into three different stages: diving, swimming and turning. During the diving stage the swimmer takes position while gliding approximately one meter under the water surface followed by a couple of strokes under water. During the swimming stage the swimmer moves actively and swims on the water surface. This causes a difference in the drag composition. At the turning point the influence of drag is inferior, because the speed is low. Just after the turn, the position is similar to the diving position.

\footnotetext{
${ }^{*}$ Corresponding author. Email: erikvangeer@gmail.com
} 


\subsection{Drag}

The total drag of a body $\left(\mathrm{F}_{\mathrm{d}}\right)$ that is gliding through the water consists of three different components [2-4]: Pressure drag $\left(\mathrm{F}_{\mathrm{p}}\right)$, wave drag $\left(\mathrm{F}_{\mathrm{w}}\right)$ and friction drag $\left(\mathrm{F}_{\mathrm{f}}\right)$. This can be written as is done in Eq. (1)

$$
F_{d}=F_{p}+F_{v}+F_{f}
$$

Friction drag depends on the surface structure of the body and the boundary layer properties in which the surface structure results. At in the used flow speed and angle. The friction drag is therefore directly related to the material used in the swimsuit. Wave drag can be seen as energy loss due to the making of waves. Energy that is used for making waves means less energy for propulsion and therefore a lower efficiency. Waves are only present while the swimmer is moving at or close to the surface. During the diving stage of the swimmer wave drag is nearly absent. The ratio between the different types of drag while swimming is not yet found and may vary from swimmer to swimmer. Generally it is assumed that pressure drag has the highest contribution to the total drag of a swimmer.

With a swimsuit there are basically two parameters that can be altered to influence drag. The material itself determines the properties of the contact surface with the water and therefore is related to friction drag. The right material can be chosen by testing different materials under similar flow-conditions in a laboratory setup, this was not part of this research.

The fit of a suit can deform the body and therefore influences the shape of the body which is related to pressure drag as well as wave drag. The FINA (Fédération Internationale de Natation) rules prohibit the use of exterior shapes on the suit and air bubbles within the suit. They state that the suit should "follow the natural human body". Different suits currently on the market have a different fit, therefore the body shape differs depending on the suit. the focus of this research project was on measuring the effect of the body shape deformation on the drag of the swimmers.

\subsection{Body shape deformation}

The ideal body shape does not create a wave behind the body, has mostly laminar flow and therefore a thinner boundary layer, and does not create any vortices around the body. The body does not have these properties naturally. Different suit brands have a different fit and therefor influence the body shape differently. This would also influence the drag characteristics differently.

\section{Method}

\subsection{Participants}

For this research it is only of interest what the performance effect is on elite swimmers. The participants are six Dutch elite female swimmers that compete in the world cup. The elite swimmers reach higher speeds that may result in different drag behavior due to the higher Reynolds number. The Reynolds number $(R n)$ depends on velocity through the water $(V)$, the body length $(l)$ and the kinetic viscosity of the fluid $(v)[1]$. This is written in Eq. (2)

$$
P_{n}=\frac{V l}{v}
$$

The swimmers also have a different body shape. In practice this means that a similar suit does not necessarily deform the body shape in the same manner between swimmers.

\subsection{Measuring methods}

To find the effect of body shape deformation on the total drag there are several parameters that need to be measured and determined. In the first place, the performance in every condition has to be measured. Next to that the body shape deformation has to be determined. The deformation is measured relative to a reference suit, in the size that the swimmer uses during competition. Because of the difficulty to define the body shape, the body is measured in two different ways: 3D scanning and traditional anthropometry measurements.

\subsubsection{Performance measuring}

The performance tests were executed by Sander Schreven at InnoSportLab Tongelreep in Eindhoven, the Netherlands. All aspects of a swimming race were tested three times per condition, diving, swimming and turning. A condition is one swimmer in one suit. To eliminate order effect the order of suits was randomized per swimmer. Before each performance test the swimmer is $3 \mathrm{D}$ scanned and measured traditionally. 


\subsubsection{D scanning}

The body shape is not only the body measurements themselves but even more the transitions over the whole body. Sharp edges or transitions generally mean that the water flow will easily turn to turbulent flow and therefore create vortices. 3D scans give insight in these details in shape transitions. The test setup is illustrated in Figure 1. The scanner is on a tripod and operated by one researcher, the computer or laptop is operated by a second researcher. The swimmer stands on a rotating platform. The scanner needs three complete rotations to gather enough information to complete the scan. This will take about 2 minutes. During the scanning period the swimmer has to stand still, a profile for the feet is used and a stick that the swimmer can hold with both hands and rest on her head. Also the swimmer is asked to stand straight and hold her position. Distractive objects are removed as much as possible to make it easier for the swimmer to concentrate. This results in a $3 \mathrm{D}$ scan of the swimmer that can be compared to the reference scan.
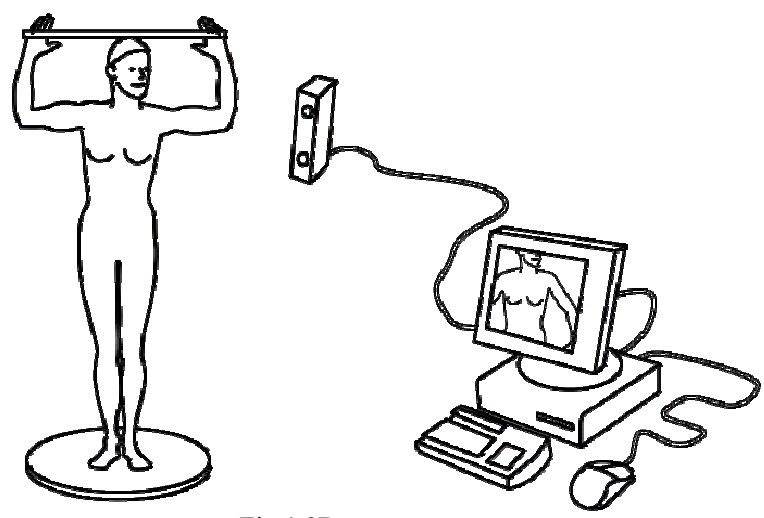

Fig 1 3D scan setup

\subsubsection{Traditional anthropometry measurements}

Next to the 3D scans also traditional anthropometry measurements are taken. In the first place to validate the accuracy of the $3 \mathrm{D}$ scans, as this is the first time that this type of scanner is used for the purpose of measuring anthropometrical sizes. The traditional measurements also give insight in the global shape of the body. It is easier to see the ratio between the different circumferences of the breast, underbreast, waist and hip in the traditional measurements.

\subsubsection{Comparing results}

Every day that tests are performed, each swimmer wearing different suits is treated as a single case. The swimmers' conditions differs from day to day. The difference in performance was measured between two or three suits, including the reference suit. This resulted in $3 \mathrm{D}$ scans and traditional measurements of the best performing suits. Using these data it can be determined if a pattern in deforming the body shape can be found for each best performing suit per individual swimmer.

\section{Results}

During this research it was the first time the Artec 3D scanner was used to measure anthropometric data. Therefore it is necessary to validate the accuracy. The results consist of 3D scans, traditional measured anthropometry measures and performance tests.

Table 1

3D scan anthropometry compared with traditional measurements, measured on one participant

\begin{tabular}{|c|c|c|c|c|c|c|c|}
\hline & $\begin{array}{l}\text { Neck circum- } \\
\text { ference }\end{array}$ & $\begin{array}{l}\text { Breast cir- } \\
\text { cumference }\end{array}$ & $\begin{array}{l}\text { Under-breast } \\
\text { circumference }\end{array}$ & $\begin{array}{l}\text { Waist cir- } \\
\text { cumference }\end{array}$ & $\begin{array}{l}\text { Hip circum- } \\
\text { ference }\end{array}$ & $\begin{array}{l}\text { Thigh cir- } \\
\text { cumference }\end{array}$ & $\begin{array}{l}\text { Knee circum- } \\
\text { ference }\end{array}$ \\
\hline Traditional (mm) & 352 & 923 & 827 & 775 & 982 & 598 & 336 \\
\hline Artec 3D (mm) & 360 & 947 & 817 & 772 & 979 & 591 & 336 \\
\hline difference $(\mathrm{mm})$ & 8 & 24 & 10 & 3 & 3 & 7 & 0 \\
\hline $\begin{array}{l}\text { Max. allowable error } \\
(\mathrm{mm}) \text {, ISO } 20685\end{array}$ & 4 & 9 & 9 & 9 & 9 & 9 & 4 \\
\hline Difference (\%) & $2,3 \%$ & $2,6 \%$ & $1,2 \%$ & $0,4 \%$ & $0,3 \%$ & $1,2 \%$ & $0,1 \%$ \\
\hline
\end{tabular}




\subsection{D scan accuracy}

The accuracy of the scanner (by the company stated as $0.2 \mathrm{~mm}$ ) was measured by collecting anthropometric data from the scans that were also measured traditionally. By comparing these data sample wise the accuracy of the 3D scanner can be assessed. These results are shown in Table 1. It striking that the breast circumferences and the neck circumference exceed the maximum allowable error that is stated in ISO 20685. This can be explained by the difference in pose. The traditional measurements are measured with the arms alongside the body while the 3D scans were made whilst the participant holding a stick above their head to stabilize their arms. This results in a different tension on their pectoralis muscles. A different tension results in a different circumference.

Next to the quantified accuracy certain flaws can occur with $3 \mathrm{D}$ scanning and should be avoided. One of these flaws is that "ghost surfaces" arise if the scan is not properly aligned. An example of this can be seen on the section view in Figure 2 within the yellow circle. Such "ghost surfaces" will give an error while measuring the circumferences. The red line in Figure 2 shows the distance over which the circumference is measured, the ghost surfaces are measured as well and add an unknown distance to the measured circumference. Ghost surfaces can be avoided through proper and precise editing of the rough scan.

Another measuring difference can be found if the circumference includes a notch. This is also illustrated in Figure 2 where a section view is shown of the hip. On the back on this scan a notch is found, the dotted yellow line illustrates what a measuring tape would measure traditionally. The red line follows exactly the shape of the back whereas a measuring tape measures the shortest circumference around the body shape. In most cases this is not an issue since the notches found are small and yields no significant difference in measurements.

Although there are some differences between the 3D scans and the traditional measurements, the differences are all explicable without questioning the accuracy of the 3D scanner. However, precise scanning and editing is necessary to acquire accurate results. The values of both measuring methods cannot directly be compared because of the different poses. This is not the purpose of the $3 \mathrm{D}$ scans during this research; the scans are not used to process circumference data but merely to visualize the smoothness of the body shape.

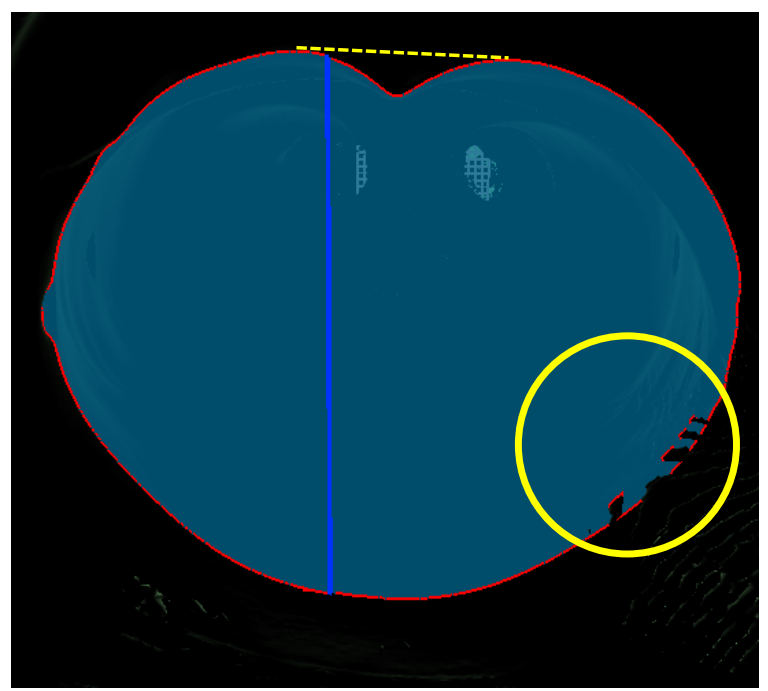

Fig. 2 A section view of the hip from a 3D scan with flaws

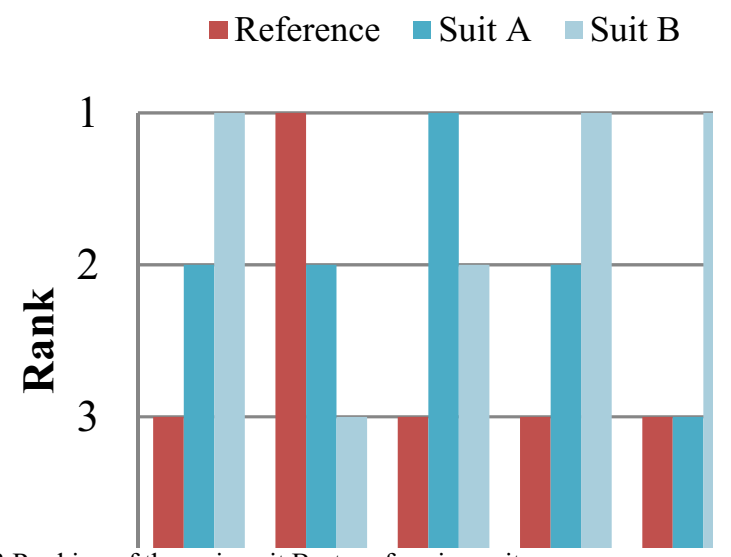

Fig. 3 Ranking of the swimsuit Best performing suits

From the drag tests, for each participant the suits can be ranked, as is shown in Figure 3, from best performing suit to least performing suit. After ranking the suits the anthropometric data and 3D scans of the best performing suit per person is compared to the least performing suit of the same participant. This shows the dominant deformation among good performing suits and may result in a pattern.

\subsection{Traditional measurements}

The measured circumferences that characterize the swimmers profile are shown in Table 2. The first column is the mean value of six swimmers after subtracting the circumferences from the $3^{\text {rd }}$ ranked suit from the highest ranked suit. As can be seen from the standard deviation, these results are insignificant and 
not accurate enough to measure the differences between the suits. The $3 \mathrm{D}$ scans can provide a solution for this

\section{Table 2}

Mean circumference differences between best and least performing suits

\begin{tabular}{|l|c|c|}
\cline { 2 - 3 } \multicolumn{1}{c|}{} & $\begin{array}{c}\text { Rank 1-Rank 3 } \\
\text { mean }(\mathrm{mm})\end{array}$ & $\begin{array}{c}\text { SD } \\
(\mathrm{mm})\end{array}$ \\
\hline breast circumference & 9,0 & 22,4 \\
\hline under-breast circumference & 6,7 & 10,4 \\
\hline waist circumference & $-14,0$ & 35,5 \\
\hline Hip circumference & 6,8 & 7,3 \\
\hline
\end{tabular}

\subsection{D scans}

3D scans can measure the entire body of a swimmer. This means that not only predetermined circumferences, but also changes in volume will be visible on the scans. Figure 4 shows the results of the scans compared to the reference suit.
In Figure 4, the scan of subject 2 had to be excluded because the scan was not accurate enough, and could not be aligned properly. The blue areas indicate places where the best performing suit dents the body more than the reference (least performing suit). Striking is the predominantly blue coloring of the scans, this suggests that compression has a positive effect on the total drag, since these are the scans of the best performing suits. The back view has a notable pattern, this is because the reference suit is an open back version whereas the best performing suits were closed back suits. Open back suits tend to push the fat towards the border of the suit on the back which results in bulging. This is most visible on the scan of subject 4 . The best performing suits are currently prohibited, because they have a zipper. Fastening features are not allowed in competition since the latest change in rules by the FINA.

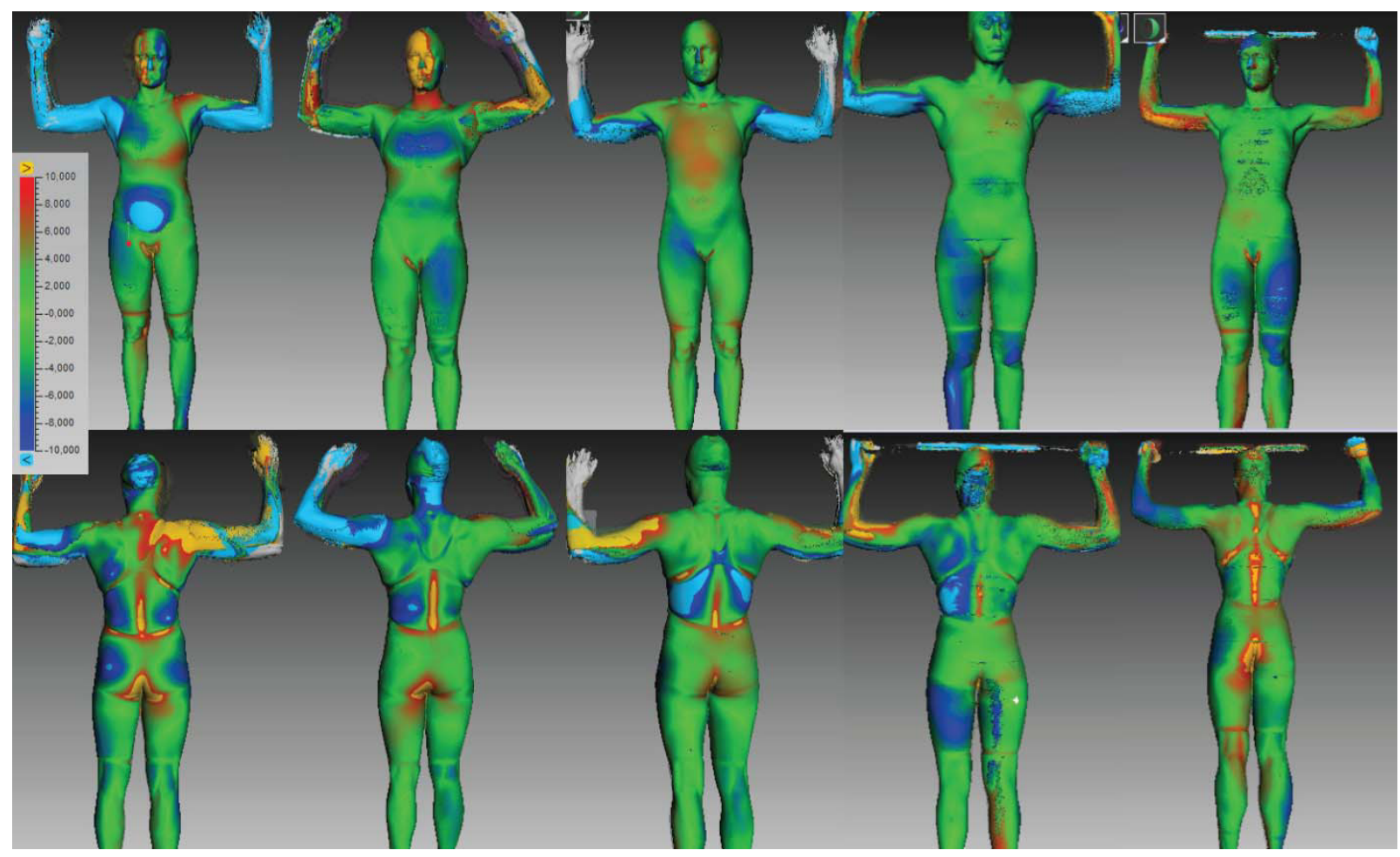

Fig. 4 3D scans of the best performing suits, respectively subjects 1, 3, 4, 5, 6 


\section{Discussion}

The subject-matter of this research is a specialist area; therefore the amount of subjects that participated was limited. Although the performance tests often show a difference in performance it is not always significant. For competitive swimmers the difference between winning and losing on the $50 \mathrm{~m}$ can be just a tenth of a second. This is within the range of $1 \%$ faster time. Because the subjects are human, it is nearly impossible to measure these differences with significance due to the natural variation a swimmer delivers over several tests.

However the different suits performed different at the performance tests. These differences were enough to make a ranking of the best performing suits. The traditional anthropometry measurements didn't yield enough information but they were useful to validate the accuracy of the 3D scanner. When comparing the deformation properties of the suits with the 3D scans, a pattern could be discerned.

Compression does seem to influence the body shape in such a way that the swimmers experience less drag. It should be kept in mind that the compression of the swimmer is not the only factor that influences the swimmers performance. The swimmers capabilities and training background is most important for good results. In the literature it is made clear that not only the shape deformation has a share in the drag reduction of the suit, but also the surface properties that cause the friction drag. There are currently no studies that convincingly show the ratio between pressure drag, friction drag and wave drag. This ratio probably varies from subject to subject. The used suits were made of similar fabric, therefore the friction drag of the suits is most likely similar. Therefore it is safe to assume that the compression as shown here, decreases drag and enhances the swimmers performance.

Because the research was performed on only six swimmers, it should be considered as a case study although it is likely that similar patters will be found using other world class swimmers.

\section{Conclusion}

3D scans give more information than can be obtained using traditional measurements. It is also easier to compare different conditions on one subject. Using a 3D scan it is possible to extract anthropometric data. The scans are most useful to compare shapes instead of data.

On these subjects, the deformation of the body shape did influence the swimming performance. The drag is reduced when using a suit that compresses. The highest ranked suits cause the body to deform in such a way that the drag is reduced. It should also be kept in mind that the reference suit in itself is often used in competition and has already a better performance than ordinary swimwear.

The deformation of the body is not the only factor that influences drag. Suits don't perform, swimmers do. The surface properties of the suit also have a share in the drag reduction. This is a different research area that remains to be explored. Next to that more research is needed to convincingly show the effect of compression on the drag reduction of swimmers.

Acknowledgement: KNZB (Royal Dutch Swimming Federation), InnoSportNL, InnoSportLab de Tongelreep

\section{References}

[1] Kuiper, G. Resistance and propulsion 1, TU Delft 2003

[2] Geer, E. van, The optimal swimsuit for forward craw, Graduation project, TU Delft, 2009.

[3] Toussaint, H. Stralen, M. van, Stevens, E. Wave drag in front crawl swimming, Institute of Fundamental and Clinical Human Movement Science, Vrije Universiteit, Amsterdam, 2002.

[4] Cuperus, N. Hijden, S. van der, Nieuwenhuijsen, K. Swimsuits and performance: The effects of manipulations to a swimsuit on passive and active drag, VU Amsterdam, 2009. 